\title{
REGULAR POINTS FOR ERGODIC SINAI MEASURES
}

\author{
MASATO TSUJII
}

\begin{abstract}
Ergodic properties of smooth dynamical systems are considered. A point is called regular for an ergodic measure $\mu$ if it is generic for $\mu$ and the Lyapunov exponents at it coincide with those of $\mu$. We show that an ergodic measure with no zero Lyapunov exponent is absolutely continuous with respect to unstable foliation [L] if and only if the set of all points which are regular for it has positive Lebesgue measure.
\end{abstract}

\section{INTRODUCTION}

We consider a dynamical system generated by a $C^{2}$ diffeomorphism $f$ on a closed $C^{2}$ manifold $M$. It is well known that the behavior of the points on $M$ under the action of $f$ is very complicated and chaotic in general and, sometimes, the orbits seem to be distributed in random way. This fact naturally leads us to the study of invariant probability measures which describe the asymptotic distribution of the orbits. Especially, we are interested in the (ergodic) measures which have attracting properties, that is, which describe the asymptotic distribution of the orbit for initial value sets with positive Lebesgue measure, because they will be observed in numerical experiments or some physical systems. The most important object in this direction is the class of measures which are called Sinai measures: invariant probability measures with no zero exponent which are absolutely continuous with respect to the unstable foliation (see [L] or $\S 4$ of this article). Ergodic Sinai measures are one generalization of the Gibbs measures on Axiom A attractors [B]. Ruelle discussed the importance of these measures in [R2], and Ledrappier studied their ergodic properties (Bernoulli property, variational relation for entropy, etc.) extensively in [L]. An important property of these ergodic measures is the fact that the set of generic points for each of them has positive Lebesgue measure [L]. Then it is natural for us to ask the question whether this attracting property characterizes ergodic Sinai measures among ergodic measures with no zero exponent. In fact, it turns out to be false. But we show, in this paper, that a littler stronger attracting property characterize ergodic Sinai measures.

Let us call a point $x \in M$ is regular for an ergodic measure $\mu_{x}$ if the following properties hold:

Received by the editors October 10, 1989.

1980 Mathematics Subject Classification (1985 Revision). Primary 58F11; Secondary 58F13. 


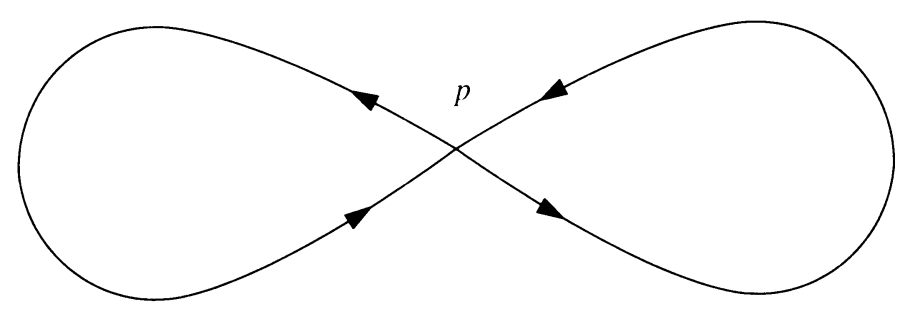

FIGURE 1

(R1) $x$ is generic for $\mu_{x}$, i.e., the sequence of measures $(1 / n) \sum_{i=0}^{n-1} \delta_{f^{i}(x)}$ converges toward $\mu_{x}$ in the sense of weak topology.

(R2) The (Lyapunov) exponents [O] at $x$ coincide with those of $\mu_{x}$ (the $\mu_{x}$-almost everywhere constant exponents).

The condition ( $\mathrm{R} 1)$ clearly means that the asymptotic distribution of the orbit $f^{i}(x), i=0,1,2, \ldots$, is described by $\mu_{x}$ and the second condition (R2) means that the asymptotic behavior of $d f$ on the tangent spaces along the orbit is regulated by $\mu_{x}$ (cf. [O]). We show the following theorems.

Theorem A. An ergodic measure $\mu$ with no zero exponent is an ergodic Sinai measure (i.e. absolutely continuous with respect to the unstable foliation) if and only if the set of all points which are regular for $\mu$ has positive Lebesgue measure.

Theorem B. Let $\mathscr{R}$ be the set of all points which are regular for ergodic measures with no zero exponent, then almost every point in $\mathscr{R}$ (w.r.t. Lebesgue measure) is regular for ergodic Sinai measures.

Remark that if we replace the condition 'regular' by 'generic', then either of the theorems is not true. The following simple but singular example shows that there exists, in general, no relation between the property of an invariant measure itself and positivity of the Lebesgue measure of the generic points for it.

Example. Let us consider a diffeomorphism on a sphere with a hyperbolic fixed point $p$ of saddle type. Suppose that the stable manifold of $p$ coincides with its unstable manifold (Figure 1 ) and $\left|\operatorname{det} d f_{p}\right|<1$. Then every point sufficiently close to the stable manifold is generic for the point measure at the saddle point $p$, which is not a Sinai measure.

In the case when we remove the condition 'ergodic,' we get the following characterization of Sinai measures.

Theorem C. For an invariant measure $\mu$ with no zero exponent, the following conditions are equivalent:

(1) $\mu$ is absolutely continuous with respect to the unstable foliation.

(2) For any Borel set $X$ with $\mu(X)>0$, the strongly stable set of $X$, $W^{s}(X)=\left\{y \in M \mid \lim \sup _{n \rightarrow+\infty}(1 / n) \log d\left(f^{n} x, f^{n} y\right)<0\right.$ for some $\left.x \in X\right\}$, has positive Lebesgue measure. 
In the course of the proof, we can get the following description for the supports of ergodic Sinai measures. This result is interesting when we compare it with some results of numerical experiments in which we can find 'strange attractors' on the closure of the unstable manifold of a hyperbolic fixed point. (See [GH, p. 91] for example.)

Proposition D. For each ergodic Sinai measure $\mu$, there exists a hyperbolic periodic point $p$ with transversal homoclinic points such that

$$
\operatorname{supp}(\mu)=\operatorname{closure}\left(W^{u}(o(p))\right)=\operatorname{closure}\left\{W^{u}(o(p)) \cap W^{s}(o(p))\right\}
$$

where $W^{s}(o(p))$ and $W^{u}(o(p))$ denote the stable and unstable manifold for the orbit of $p$, respectively.

This paper proceeds as follows: In $\S 2$ and $\S 3$, we give some basic estimations and a little modification of known results in [P], [R1] and [PS]. In $\S 4$, we construct ergodic measures for which almost every point in $\mathscr{R}$ are regular, and show that they are ergodic Sinai measures. We prove the main results at the end of $\S 4$.

Before we go into the proof, let us give some remarks on the notations. We will denote the set of nonnegative integer by $\mathbf{Z}^{+}$. The symbol $\varphi: X \nearrow Y$ means that $\varphi$ is a mapping from a subset $\mathscr{D}(f)$ of $X$ to $Y$. For two mappings $f: X \nearrow Y$ and $g: Y \nearrow Z, g \circ f: X \nearrow Z$ denotes the composition of $f$ and $g$ defined on $\mathscr{D}(g \circ f)=\mathscr{D}(f) \cap f^{-1}(\mathscr{D}(g))$. When $f: X \nearrow Y$ is injective on $\mathscr{D}(f), f^{-1}$ denotes the inverse map defined on $\mathscr{D}\left(f^{-1}\right)=f(\mathscr{D}(f))$.

The author would like to thank Professor Masahisa Adachi for his warm encouragment to this work.

\section{BASIC ESTIMATIONS}

In this section, we collect some preparatory estimations for the behavior of the diffeomorphism on tangent spaces. We fix a Riemannian metric $\langle\cdot, \cdot\rangle_{x}$ on $M$, and denote the corresponding norm and distance by $\|\cdot\|_{x}$ and $d(\cdot, \cdot)$, respectively. We also fix a positive number $\varepsilon \in\left(0,10^{-2}\right)$ throughout this paper. First of all, let us introduce subsets $\Lambda_{\chi, l}^{k}$ and $\Gamma_{\chi, l}^{k}\left(k \in \mathbf{Z}^{+}, 0<\right.$ $\left.\chi<10^{-1}, 1 \leq l\right)$ which play important rolls in the study of measures with no zero exponent (cf. [P1], [P2], [K]): $\Lambda_{\chi, l}^{k}\left(\right.$ resp. $\left.\Gamma_{\chi, l}^{k}\right)$ is the set of all points $x \in M$ at which there exists a decomposition of tangent space $T_{x} M=E_{x}^{s} \oplus E_{x}^{u}$ with $\operatorname{dim} E_{x}^{s}=k$ satisfying the following conditions (1), (2) and (3) for every $n \in \mathbf{Z}^{+}, m \in \mathbf{Z}$ (resp. every $n \in \mathbf{Z}^{+}, m \in \mathbf{Z}^{+}$)

(1) $\left\|d f^{n} u\right\| \leq(l \cdot \exp (\varepsilon \chi|m|)) \cdot \exp (-\chi n)\|u\|$ for every $u \in d f_{x}^{m} E_{x}^{s}$,

(2) $\left\|d f^{n} v\right\| \geq(l \cdot \exp (\varepsilon \chi|m|))^{-1} \cdot \exp (\chi n)\|v\|$ for every $v \in d f_{x}^{m} E_{x}^{u}$,

(3) $\gamma(x, m) \geq(l \cdot \exp (\varepsilon \chi|m|))^{-1}$ where $\gamma(x, m)$ denote the angle between $d f_{x}^{m} E_{x}^{s}$ and $d f_{x}^{m} E_{x}^{u}$.

Put

$$
\tilde{\Lambda}_{\chi, l}^{k}=\left\{x \in M \mid m^{\prime}\left(\left\{n \in \mathbf{Z}^{+} \mid f^{n} x \in \Lambda_{\chi, l}^{k}\right\}\right)>0\right\}
$$


and

$$
\widetilde{\Gamma}_{\chi, l}^{k}=\left\{x \in M \mid m^{\prime}\left(\left\{n \in \mathbf{Z}^{+} \mid f^{n} x \in \Gamma_{\chi, l}^{k}\right\}\right)>0\right\}
$$

where $m^{\prime}(B)=\liminf _{n \rightarrow \infty}(1 / n) \#\{B \cap\{0,1,2, \ldots, n-1\}\}$. We can see the following properties of the sets $\Lambda_{\chi, l}^{k}, \Gamma_{\chi, l}^{k}$ and the decomposition $T_{x} M=$ $E_{x}^{s} \oplus E_{x}^{u}$ in their definitions (cf. [P1]).

Lemma 1. (1) $\Lambda_{\chi, l}^{k} \subset \Gamma_{\chi, l}^{k}$ and $\Lambda_{\chi, l}^{k} \subset \Lambda_{\chi, l^{\prime}}^{k}, \Gamma_{\chi, l}^{k} \subset \Gamma_{\chi, l^{\prime}}^{k}$ for $l \leq l^{\prime}$.

(2) $f\left(\Lambda_{\chi, l}^{k}\right) \cup f^{-1}\left(\Lambda_{\chi, l}^{k}\right) \subset \Lambda_{\chi, l \cdot \exp (\varepsilon \chi)}^{k}$ and $f\left(\Gamma_{\chi, l}^{k}\right) \subset \Gamma_{\chi, l \cdot \exp (\varepsilon \chi)}^{k}$.

(3) $\Lambda_{\chi, l}^{k}$ and $\Gamma_{\chi, l}^{k}$ are compact sets.

(4) On each $\Lambda_{\chi, l}^{k}$, the subspaces $E_{x}^{s}$ and $E_{x}^{u}$ are uniquely determined and depend on $x$ continuously.

(5) On each $\Gamma_{\chi, l}^{k}$, the subspace $E_{x}^{s}$ is uniquely determined and depends on $x$ continuously.

The following important lemma is due to Pesin [P1].

Lemma 2. Let $y \in M$ be a regular point (resp. forward regular point) in the sense of Oseledec's theorem (see [P1] for definitions) and the exponents $\lambda_{i}(y)$, $i=1,2, \ldots, \operatorname{dim} M$ satisfy, for $\chi \in\left(0,10^{-1}\right)$ and $k \in \mathbf{Z}^{+}$,

$$
\begin{aligned}
\lambda_{1}(y) & \leq \lambda_{2}(y) \leq \cdots \leq \lambda_{k}(y)<-(1+\varepsilon) \chi<0<(1+\varepsilon) \chi \\
& <\lambda_{k+1}(y) \leq \cdots \leq \lambda_{\operatorname{dim} M}(y),
\end{aligned}
$$

then $y$ belongs to $\Lambda_{\chi, l}^{k}\left(\right.$ resp. $\left.\Gamma_{\chi, l}^{k}\right)$ for some $l \geq 1$.

For the points in the set $\mathscr{R}$, we have the following estimation.

Proposition 3. If a point $y \in \mathscr{R}$ satisfies (1) for some $\chi$ and $k$, then $y$ is contained in the set $\bigcup_{l \geq 1} \tilde{\Gamma}_{\chi^{\prime}, l}^{k}$ where $\chi^{\prime}=(1-2 \varepsilon) \chi$.

For the proof, we need an elementary lemma.

Lemma 4. For $\delta \in(0,1 / 4)$, let $B$ be a subset of $\mathbf{N}$ satisfying $m^{\prime}(B)>\left(1-\delta^{2}\right)$ and let $\mathscr{B}$ be the set of all intervals $I=\{n, n+1, \ldots, n+k-1\}$ in $\mathbf{N}$ which satisfies $(1 / k) \cdot \#(I-B)>\delta$, then we have $m^{\prime}\left(\mathbf{N}-\bigcup_{I \in \mathscr{B}} I\right)>1-5 \delta$.

Proof. Take a large number $N$ so that, for every $n \geq N$,

$$
(1 / n) \#\{B \cap\{1,2, \ldots, n\}\}>1-\delta^{2}
$$

and set

$$
\mathscr{B}_{n}=\{I \in \mathscr{B} \mid I \subset\{1,2, \ldots, n\}\} .
$$

Then we have, for $n \geq N$,

$$
\left(\bigcup_{I \in \mathscr{B}_{n}} I\right) \cup\{n-[\delta n], n-[\delta n]+1, \ldots, n\} \supset\left(\bigcup_{I \in \mathscr{B}} I\right) \cap\{1,2, \ldots, n\}
$$


where [.] is Gauss's symbol. Choose a sequence of intervals

$$
I_{i}=\left\{n_{i}, n_{i}+1, \ldots, n_{i}+k_{i}-1\right\} \in \mathscr{B}_{n}
$$

and

$$
J_{i}=\left\{n_{i}-k_{i}, n_{i}-k_{i}+1, \ldots, n_{i}+2 k_{i}-1\right\},
$$

$i=1,2, \ldots, i(n)$ inductively so that $I_{i}$ has the maximal length among the intervals in $\mathscr{B}_{n}$ which are not completely contained in $\bigcup_{j=1}^{i-1} J_{j}$ and $\left(\bigcup_{j=1}^{i(n)} J_{j}\right) \supset$ $\left(\bigcup_{I \in \mathscr{B}_{n}} I\right)$. Since the intervals $I_{i}$ are disjoint each other, we have

$$
\#\left(\bigcup_{I \in \mathscr{B}_{n}} I\right) \leq \#\left(\bigcup_{j=1}^{i(n)} J_{j}\right) \leq 3 \cdot \#\left(\bigcup_{j=1}^{i(n)} I_{j}\right) \leq 3[\delta n] .
$$

Therefore $\#\left(\{1,2, \ldots, n\} \cap\left(\bigcup_{I \in \mathscr{B}} I\right)\right) \leq 4 \delta n+1$ for $n \geq N$.

Proof of Proposition 3. From the condition (R2), we can see that the point $y$ is a forward regular point (cf. [O]) and, from Lemma 2, $y \in \Gamma_{\chi, l}^{k}$ for some $l \geq 1$. Let us fix a decomposition $T_{y} M=E_{y}^{s} \oplus E_{y}^{u}$ in the definition of $\Gamma_{\chi, l}^{k}$, and define functions $\rho^{s}, \rho^{u}, \rho^{\gamma}, l^{s}, l^{u}$ and $l^{\gamma}: \mathbf{Z}^{+} \rightarrow \mathbf{R}$ by

$$
\begin{aligned}
& \rho^{s}(n)=\sup _{k \in \mathbf{Z}^{+}}\left\{\left\|\left.d f^{k}\right|_{d f^{n}\left(E_{y}^{s}\right)}\right\| \cdot \exp ((1-2 \varepsilon) \chi k)\right\}, \\
& \rho^{u}(n)=\sup _{k \in \mathbf{Z}^{+}}\left\{\left\|\left.d f^{-k}\right|_{d f^{n+k}\left(E_{y}^{u}\right)}\right\| \cdot \exp ((1-2 \varepsilon) \chi k)\right\}, \\
& \rho^{\gamma}(n)=\left(\text { angle between } d f^{n} E_{y}^{s} \text { and } d f^{n} E_{y}^{u}\right)
\end{aligned}
$$

and

$$
l^{j}(n)=\sup _{k \in \mathbf{Z}^{+}}\left\{\rho^{j}(n+k) \cdot \exp (-\varepsilon(1-2 \varepsilon) \chi k)\right\}, \quad j=s, u, \gamma .
$$

Then we have, for $R \geq 1$,

$$
\left\{n \in \mathbf{Z}^{+} \mid f^{n}(y) \in \Gamma_{\chi^{\prime}, R}^{k}\right\} \supset\left\{n \in \mathbf{Z}^{+} \mid \max \left(l^{s}(n), l^{u}(n), l^{\gamma}(n)\right) \leq R\right\} .
$$

In order to prove the proposition, we shall show, for some large $R$,

$$
m^{\prime}\left(\left\{n \in \mathbf{Z}^{+} \mid l^{j}(n) \leq R\right\}\right) \geq 9 / 10 \quad \text { for } j=s, u, \gamma .
$$

Put $K=\max \left\{\mid \log \|d f(v)\|-\log \|v\| \| 0 \neq v \in T_{x} M, x \in M\right\}$ and $\eta=$ $(1 / 5)(\varepsilon(1-2 \varepsilon) \chi / 2 K)^{2}$. From Lemma 2 and Oseledec's theorem [O] we have $\mu_{y}\left(\bigcup_{l} \Lambda_{\chi, l}^{k}\right)=1$ and, therefore, we can take a number $r \geq 1$ so that $\mu_{y}\left(\Lambda_{\chi, r}^{k}\right)>$ $1-\eta^{2}$.

Let us consider the Grassmannian bundle $G^{k}(M)$ over $M$ which consists of $k$-dimensional subspaces of tangent spaces, and take a neighborhood $U$ of the compact set $\left(\bigcup_{x \in \Lambda_{x, r}^{k}} E_{x}^{s}\right) \subset G^{k}(M)$ sufficiently small so that every tangent vector $u$ in $E \in U$ satisfies

$$
\left\|d f^{n} u\right\|<2 r \cdot \exp (-\chi n)\|u\| \quad \text { for } 0 \leq n \leq 2 N_{0},
$$


where $N_{0}=\left[(\log 2 r)(\varepsilon \chi)^{-1}\right]+1$. From the condition (R2), we can see that $m^{\prime}\left(\left\{n \in \mathbf{N} \mid d f^{n}\left(E_{y}^{s}\right) \in U\right\}\right)>1-\eta^{2}$. Next we shall show that, if $\rho^{s}(n)>$ $\exp \left(4 K N_{0}\right)$, then there exists $p \in \mathbf{N}$ such that

$$
\begin{gathered}
\left(1 / p N_{0}\right) \#\left(\left\{i \in \mathbf{Z}^{+} \mid d f^{i}\left(E_{y}^{s}\right) \in U\right\} \cap\left\{n, n+1, \ldots, n+p N_{0}-1\right\}\right) \\
<1-(\varepsilon \chi / 2 K)<1-\eta .
\end{gathered}
$$

By assumption, there exist a number $k=p \cdot N_{0}+q>0,\left(p, q \in \mathbf{Z}^{+}, q<N_{0}\right)$ and a tangent vector $0 \neq u \in d f^{n} E_{y}^{s}$ for which we have

$$
\left\|d f^{k} u\right\|>\exp \left(4 K N_{0}\right) \cdot \exp (-(1-2 \varepsilon) \chi k)\|u\| .
$$

For $0 \leq i<p$, set

$$
\begin{aligned}
& P_{i}=1 \quad \text { if there exists a number } m(i) \in\left\{i N_{0}, i N_{0}+1, \ldots,(i+1) N_{0}-1\right\} \\
& \text { such that } d f^{n+m(i)}\left(E_{y}^{s}\right) \in U,
\end{aligned}
$$

$P_{i}=0$ otherwise. (In this case, we set $m(i)=(i+1) N_{0}-1$.)

Put $m(p)=p \cdot N_{0}$. Then we have, for $u \in d f^{n} E_{y}^{s}$, $\left\|d f^{k} u\right\| /\|u\| \leq \exp \left(2 K N_{0}\right) \cdot\left\|\left.d f^{m(p)-m(0)}\right|_{d f^{n+m(0)} E_{y}^{s}}\right\|$

$$
\begin{aligned}
& \leq \exp \left(2 K N_{0}+\sum_{i=0}^{p-1}\left\{\log (2 r) \cdot P_{i}-\chi\{m(i+1)-m(i)\} \cdot P_{i}\right.\right. \\
& \left.\left.\qquad K\{m(i+1)-m(i)\}\left(1-P_{i}\right)\right\}\right) \\
& \leq \exp \left(2 K N_{0}+p \cdot \log (2 r)\right. \\
& \left.\quad-\chi\{m(p)-m(0)\}+(K+\chi) N_{0} \sum_{i=0}^{p-1}\left(1-P_{i}\right)\right) \\
& \leq \exp \left(4 K N_{0}+\left(k / N_{0}\right) \cdot \log (2 r)-\chi k+2 K N_{0} \sum_{i=0}^{p-1}\left(1-P_{i}\right)\right) \\
& \leq \exp \left(4 K N_{0}-\varepsilon \chi k+2 K N_{0} \sum_{i=0}^{p-1}\left(1-P_{i}\right)\right) \cdot \exp (-(1-2 \varepsilon) \chi k)\|u\| .
\end{aligned}
$$

This implies (3). Applying Lemma 4, we have

$$
m^{\prime}\left(\left\{n \in \mathbf{N} \mid \rho^{s}(n) \leq \exp \left(4 K N_{0}\right)\right\}\right)>1-5 \eta=1-(\varepsilon(1-2 \varepsilon) \chi / 2 K)^{2} .
$$

Since $\rho^{s}(n+1) \leq \exp (K) \cdot \rho^{s}(n)$, we can see that, if $l^{s}(n)>\exp \left(4 K N_{0}\right)$, there exists $k \in \mathbf{Z}^{+}$such that

$(1 / k) \#\left(\{n, n+1, \ldots, n+k-1\} \cap\left\{j \mid \rho^{s}(j)>\exp \left(4 K N_{0}\right)\right\}\right)>\varepsilon(1-2 \varepsilon) \chi / 2 K$.

Again by Lemma 4, we have

$$
m^{\prime}\left(\left\{n \in \mathbf{Z}^{+} \mid l^{s}(n)<\exp \left(4 K N_{0}\right)\right\}\right)>1-5(\varepsilon(1-2 \varepsilon) \chi / 2 K)>9 / 10 .
$$

Similarly, we can show (2) $j=u, \gamma$ for sufficiently large $R$. 


\section{IMPROVEMENT OF LYAPUNOV METRIC}

In this section, we fix $\chi \in\left(0,10^{-1}\right), k \in \mathbf{Z}^{+}$and denote $\Lambda_{\chi, l}^{k}=\Lambda_{l}$, $\Gamma_{\chi, l}^{k}=\Gamma_{l}, \bigcup_{l} \Lambda_{l}=\Lambda$ and $\bigcup_{l} \Gamma_{l}=\Gamma$, simply. We define a measurable Riemannian metric $\langle\cdot, \cdot\rangle_{x}^{\prime \prime}$ on $\Lambda$, which is sometimes called Lyapunov metric, in the following way: for $v_{i}=v_{i}^{s}+v_{i}^{u}\left(v_{i}^{s} \in E_{x}^{s}, v_{i}^{u} \in E_{x}^{u}, i=1,2\right)$,

$$
\left\langle v_{1}, v_{2}\right\rangle_{x}^{\prime \prime}=\left\langle v_{1}^{s}, v_{2}^{s}\right\rangle_{x}^{\prime \prime}+\left\langle v_{1}^{u}, v_{2}^{u}\right\rangle_{x}^{\prime \prime}
$$

where

$$
\left\langle v_{1}^{s}, v_{2}^{s}\right\rangle^{\prime \prime}=\sum_{k=0}^{\infty}\left\langle d f_{x}^{k} v_{1}^{s}, d f_{x}^{k} v_{2}^{s}\right\rangle \cdot \exp (2(1-2 \varepsilon) \chi k)
$$

and

$$
\left\langle v_{1}^{u}, v_{2}^{u}\right\rangle^{\prime \prime}=\sum_{k=0}^{\infty}\left\langle d f_{x}^{-k} v_{1}^{u}, d f_{x}^{-k} v_{2}^{u}\right\rangle^{\prime \prime} \cdot \exp (2(1-2 \varepsilon) \chi k) .
$$

The corresponding norm we denote by $\|\cdot\|_{x}^{\prime \prime}$. This metric has the following properties (cf. [P1]):

$\left(\mathrm{a}^{\prime}\right)$ we have, for every $u \in E_{x}^{s}$,

$$
\exp (-K) \leq\left\|d f_{x}(u)\right\|_{f x}^{\prime \prime} /\|u\|_{x}^{\prime \prime} \leq \exp (-(1-2 \varepsilon) \chi k)
$$

and, for every $v \in E_{x}^{u}$,

$$
\exp ((1-2 \varepsilon) \chi) \leq\left\|d f_{x}(v)\right\|_{f x}^{\prime \prime} /\|v\|_{x}^{\prime \prime} \leq \exp (K)
$$

where $K=\max \left\{\mid \log \|d f(v)\|-\log \|v\| \| v \in T_{x} M, x \in M\right\}$.

$\left(b^{\prime}\right)$ on each $\Lambda_{l}(l \geq 1),\langle\cdot, \cdot\rangle_{x}^{\prime \prime}$ depends on $x$ continuously.

(c') $(1 / 2)\|\cdot\|_{x} \leq\|\cdot\|_{x}^{\prime \prime} \leq 4 \cdot l^{2} \cdot(1-\exp (-2 \varepsilon \chi))^{-1 / 2}\|\cdot\|_{x}$ for $x \in \Lambda_{l}$. From this metric $\|\cdot\|^{\prime \prime}$, we construct another metric $\|\cdot\|_{x}^{\prime}$ on $\Lambda$ with the properties that enable us to apply the techniques for uniformly hyperbolic sets to the set $\Lambda$. Let us define a function $l: \Lambda \rightarrow \mathbf{R}$ by $l(x)=\min \left\{l \geq 1 \mid x \in \Lambda_{l}\right\}$. Then it satisfies, for $m \in \mathbf{Z}$ and $x \in \Lambda$,

$$
l\left(f^{m} x\right) \leq l(x) \exp (\varepsilon \chi|m|) .
$$

Lemma 5. There exists a function $A: \Lambda \rightarrow \mathbf{R}$ which is continuous on each $\Lambda_{l}$ $(l \geq 1)$ and satisfies, for every $x \in \Lambda$ and $m \in \mathbf{Z}$,

$$
C \cdot l^{2}(x) \geq A(x) \geq \max \left\{\|v\|_{x}^{\prime \prime} /\|v\|_{x} \mid v \in T_{x} M\right\}
$$

and $A\left(f^{m} x\right) \leq A(x) \cdot \exp (4 \varepsilon \chi|m|)$ where $C$ is a constant.

Proof. Put $\widetilde{A}^{j}(x)=\sup \left\{\|v\|_{x}^{\prime \prime} /\|v\|_{x} \mid v \in E_{x}^{j}-0\right\} \quad(j=s, u)$ and $\widetilde{A}^{\gamma}(x)=$ (angle between $E_{x}^{s}$ and $\left.E_{x}^{u}\right)^{-1}$. Then $\widetilde{A}^{j}(x)(j=s, u, \gamma)$ is continuous on each $\Lambda_{l}$, and satisfies, for some constant $C>4, \tilde{A}^{j}(x) \leq(\sqrt{C} / 2) l(x)$. Put

$$
\begin{aligned}
A^{j}(x) & =\max \left\{1, \max \left\{\widetilde{A}^{j}\left(f^{k} x\right) \exp (-2 \varepsilon \chi|k|) \mid k \in \mathbf{Z}\right\}\right\} \\
& =\max \left\{1, \max \left\{\widetilde{A}^{j}\left(f^{k} x\right) \exp (-2 \varepsilon \chi|k|)|| k \mid \leq \log (\sqrt{C} l(x)) / \varepsilon \chi\right\}\right\},
\end{aligned}
$$

then $A^{j}(x)$ is continuous on each $\Lambda_{l}$ and satisfies, for $x \in \Lambda$ and $m \in \mathbf{Z}$, 
$A^{j}\left(f^{m} x\right) \leq A^{j}(x) \cdot \exp (2 \varepsilon \chi|m|)$ and $\tilde{A}^{j}(x) \leq A^{j}(x) \leq(\sqrt{C} / 2) \cdot l(x)$. It is easy to check that the function $A(x)=4 \cdot A^{\gamma}(x) \cdot \max \left(A^{s}(x), A^{u}(x)\right)$ satisfies all the conditions.

We denote, by $\exp _{x}: T_{x} M \nearrow M$, the exponential mapping at $x \in M$ defined on $\mathscr{D}\left(\exp _{x}\right)=\left\{v \in T_{x} M \mid\|v\|<r_{0}\right\}$ where $r_{0}$ is the injectivity radius, and set

$$
f_{x}=\left(\exp _{x}\right)^{-1} \circ f \circ\left(\exp _{x}\right): T_{x} M \nearrow T_{f x} M .
$$

Since $M$ is compact, we can find constants $\alpha=\alpha(f)>0$ and $\beta=\beta(f)>0$ satisfying the following conditions, for every $x \in M$,

(1) $\mathscr{D}\left(f_{x}\right) \supset D(x, \alpha) \equiv\left\{v \in T_{x} M \mid\|v\| \leq \alpha\right\}$,

(2) for every $w, z \in D(x, \alpha)$, we have

$$
(1 / 2)\|w-z\| \leq d\left(\exp _{x} w, \exp _{x} z\right) \leq 2\|w-z\|
$$

and

$$
\left\|d\left(f_{x}\right)_{w}-d\left(f_{x}\right)_{z}\right\| \leq \beta\|w-z\|
$$

where we denote the operator norm with respect to the norm $\|\cdot\|$ by the same symbol $\|\cdot\|$.

Proposition 6. For any $D>0$, there exists a measurable Riemannian metric $\langle\cdot, \cdot\rangle_{x}^{\prime}$ and the corresponding norm $\|\cdot\|_{x}^{\prime}$ on $\Lambda$ with the following properties:

(a) $\exp (-K-4 \varepsilon \chi) \leq\left\|d f_{x}(u)\right\|_{f x}^{\prime} /\|u\|_{x}^{\prime} \leq \exp (-(1-6 \varepsilon) \chi)$ for every $u \in E_{x}^{s}$ and $\exp ((1-6 \varepsilon) \chi) \leq\left\|d f_{x}(v)\right\|_{f x}^{\prime} /\|v\|_{x}^{\prime} \leq \exp (K+4 \varepsilon \chi)$ for every $v \in E_{x}^{u}$.

(b) On each $\Lambda_{l}(l \geq 1)$, the metric $\langle\cdot, \cdot\rangle_{x}^{\prime}$ depend on $x$ continuously.

(c) $\|\cdot\|_{x} \leq\|\cdot\|_{x}^{\prime} \leq C_{D} \cdot l(x)^{4}\|\cdot\|_{x}$ where $C_{D}$ is a constant depending on $D$.

(d) For every $w, z \in D(x, \alpha)$, we have $\left\|d\left(f_{x}\right)_{w}-d\left(f_{x}\right)_{z}\right\|^{\prime} \leq D\|w-z\|_{x}^{\prime}$ where we denote the operator norm with respect to $\|\cdot\|_{x}^{\prime}$ and $\|\cdot\|_{f x}^{\prime}$ by the symbol $\|\cdot\|^{\prime}$.

(e) $D(x, \alpha) \supset\left\{(v, u) \in E_{x}^{s} \oplus E_{x}^{u}=T_{x} M \mid\|u\|^{\prime} \leq \kappa,\|v\|^{\prime} \leq \kappa\right\}$ where $\kappa=\exp (K+1)$.

(f) $E_{x}^{s}$ and $E_{x}^{u}$ are orthogonal with respect to $\langle\cdot, \cdot\rangle^{\prime}-x$.

Remark. Much the same type of metric can be found in Pugh and Shub's recent work [PS, 3.8]. But we give the proof of this proposition here because we need the continuous dependence of the metric $\|\cdot\|_{x}^{\prime}$ on $x$ (which is not stated in [PS]) and because we will treat the metrics on the set $\Gamma$ in the parallel way.

Proof. Let $A(x)$ be the function which we constructed in Lemma 5, and set $\|\cdot\|^{\prime}=c \cdot A(x)\|\cdot\|^{\prime \prime}$ for $c>1$. Then we have, for $w, z \in D(x, \alpha)$,

$$
\begin{aligned}
\left\|d\left(f_{x}\right)_{w}-d\left(f_{x}\right)_{z}\right\|^{\prime} & =A(f x) A(x)^{-1}\left\|d\left(f_{x}\right)_{w}-d\left(f_{x}\right)_{z}\right\|^{\prime \prime} \\
& \leq 2 \beta \cdot A(f x)^{2} A(x)^{-1}\|w-z\| \\
& \leq 4 \beta \cdot c^{-1} \cdot A(f x)^{2} A(x)^{-2}\|w-z\|^{\prime} \\
& \leq 4 \beta \cdot c^{-1} \cdot \exp (8 \varepsilon \chi)\|w-z\|^{\prime} .
\end{aligned}
$$


Therefore, if we take sufficiently large $c$, then $\|\cdot\|^{\prime}$ satisfy the condition (d). We can check the other conditions easily.

Next we construct a family of metrics on $\Gamma$ which has the properties similar to $\|\cdot\|^{\prime}$ on $\Lambda$. We denote, by $\left(E_{z}^{s}\right)^{\perp}$, the orthogonal complement for $E_{z}^{s}$ with respect to the inner product $\langle\cdot, \cdot\rangle_{z}$ for $z \in \Gamma$. Let us define an inner product $\langle\cdot, \cdot\rangle_{z, n}^{\prime \prime}$ on $T_{f^{n} z} M$ for $z \in \Gamma$ and $n \in \mathbf{Z}^{+}$in the following way: for $v_{j}=v_{j}^{s}+v_{j}^{t}\left(v_{j}^{s} \in d f^{n} E_{z}^{s}, v_{j}^{t} \in d f^{n}\left(\left(E_{z}^{s}\right)^{\perp}\right), j=1,2\right)$

$$
\left\langle v_{1}, v_{2}\right\rangle_{z, n}^{\prime \prime}=\left\langle v_{1}^{s}, v_{2}^{s}\right\rangle_{z, n}^{\prime \prime}+\left\langle v_{1}^{t}, v_{2}^{t}\right\rangle_{z, n}^{\prime \prime}
$$

where

$$
\left\langle v_{1}^{s}, v_{2}^{s}\right\rangle_{z, n}^{\prime \prime}=\sum_{k=0}^{\infty}\left\langle d f^{k} v_{1}^{s}, d f^{k} v_{2}^{s}\right\rangle \cdot \exp (2(1-2 \varepsilon) \chi k)
$$

and

$$
\left\langle v_{1}^{t}, v_{2}^{t}\right\rangle_{z, n}^{\prime \prime}=\sum_{k=0}^{n}\left\langle d f^{-k} v_{1}^{t}, d f^{-k} v_{2}^{t}\right\rangle \cdot \exp (2(1-2 \varepsilon) \chi k)
$$

The corresponding norm we denote by $\|\cdot\|_{z, n}^{\prime \prime}$. Then it has the following properties:

$\left(\mathrm{a}^{\prime \prime}\right)$ we have, for every $u \in d f^{n}\left(E_{z}^{s}\right), n \geq 0$,

$$
\exp (-K) \leq\|d f(u)\|_{z, n+1}^{\prime \prime} /\|u\|_{z, n}^{\prime \prime} \leq \exp (-(1-2 \varepsilon) \chi)
$$

and, for every $v \in d f^{n}\left(\left(E_{z}^{s}\right)^{\perp}\right), n \geq 0$,

$$
\exp ((1-2 \varepsilon) \chi) \leq\|d f(u)\|_{z, n+1}^{\prime \prime} /\|u\|_{z, n}^{\prime \prime} \leq \exp (K),
$$

$\left(\mathbf{b}^{\prime \prime}\right)$ on each $\Gamma_{l}(l \geq 1),\langle\cdot, \cdot\rangle_{z, 0}^{\prime \prime}$ depends on $z$ continuously.

$\left(\mathrm{c}^{\prime \prime}\right)(1 / 2)\|\cdot\|_{f^{n} z} \leq\|\cdot\|_{z, n}^{\prime \prime} \leq 8 l^{4} \cdot \exp (2 \varepsilon \chi n) \cdot\{1-\exp (-4 \varepsilon \chi)\}^{-1 / 2}\|\cdot\|_{f^{n_{z}}}$ for $z \in \Gamma_{l}$ and $n \geq 0$.

The properties $\left(a^{\prime \prime}\right)$ and $\left(b^{\prime \prime}\right)$ can be checked easily. But, in this case, the property $\left(c^{\prime \prime}\right)$ is not so obvious. We prove it here. Let us fix a decomposition $T_{z} M=E_{z}^{s} \oplus E_{z}^{u}$ satisfying the conditions in the definition of $\Gamma_{l}$. For any $v \in T_{f^{n} z} M \quad(n \geq 0)$, we consider a decomposition $v=v^{s}+v^{t s}+v^{t u}$ such that $v^{s}, v^{t s} \in d f^{n}\left(E_{z}^{s}\right), v^{t u} \in d f^{n}\left(E_{z}^{u}\right)$ and $v^{t}=v^{t s}+v^{t u} \in d f^{n}\left(\left(E_{z}^{s}\right)^{\perp}\right)$. Then we have

$$
\begin{gathered}
\left\|v^{t u}\right\| \leq(\sin \gamma(z, n))^{-1}\|v\| \leq(2 l \cdot \exp (\varepsilon \chi n))\|v\|, \\
\left\|v^{s}+v^{t s}\right\| \leq(2 l \cdot \exp (\varepsilon \chi n))\|v\|
\end{gathered}
$$


and, for $0 \leq k \leq n$,

$$
\begin{aligned}
\left\|d f^{-k} v^{t u}\right\| & \leq l \cdot \exp (\varepsilon \chi(n-k)) \cdot \exp (-\chi k)\left\|v^{t u}\right\| \\
& \leq 2 l^{2} \cdot \exp (\varepsilon \chi(2 n-k)) \cdot \exp (-\chi k)\|v\|, \\
\left\|d f^{-k} v^{t s}\right\| & \leq l \cdot \exp (-\chi(n-k))\left\|d f^{-n} v^{t s}\right\| \\
& \leq l \cdot \exp (-\chi(n-k))\left\|d f^{-n} v^{t u}\right\| \\
& \leq 2 l^{3} \cdot \exp (\varepsilon \chi n) \cdot \exp (-\chi n)\|v\|
\end{aligned}
$$

Combining these, we can get, for $0 \leq k \leq n$,

$$
\left\|d f^{-k} v^{t}\right\| \leq 4 l^{3} \cdot \exp (2 \varepsilon \chi n) \exp (-\chi k)\|v\|
$$

and, for $0 \leq k$,

$$
\begin{aligned}
\left\|d f^{k} v^{s}\right\| & \leq l \cdot \exp (\varepsilon \chi n) \cdot \exp (-\chi k) \cdot\left(\left\|v^{s}+v^{t s}\right\|+\left\|v^{t s}\right\|\right) \\
& \leq 4 l^{4} \cdot \exp (2 \varepsilon \chi n) \exp (-\chi k)\|v\| .
\end{aligned}
$$

Therefore,

$$
\left\|v^{l}\right\|_{z, n}^{\prime \prime} \leq 4 l^{3} \cdot \exp (2 \varepsilon \chi n)\{1-\exp (-4 \varepsilon \chi)\}^{-1 / 2}\|v\|
$$

and

$$
\left\|v^{s}\right\|_{z, n}^{\prime \prime} \leq 4 l^{4} \cdot \exp (2 \varepsilon \chi n)\{1-\exp (-4 \varepsilon \chi)\}^{-1 / 2}\|v\|,
$$

and we get the right inequality of $\left(c^{\prime \prime}\right)$. The left inequality can be seen easily.

Proposition 7. For any positive number $D$, there exists a family of inner products

$$
\left\{\langle\cdot, \cdot\rangle_{z, n}^{\prime}: T_{f} n_{z} M \times T_{f} n_{z} M \rightarrow \mathbf{R} \mid z \in \Gamma, n \in \mathbf{Z}^{+}\right\}
$$

satisfying the following conditions:

(a) $\exp (-K-6 \varepsilon \chi) \leq\|d f(u)\|_{z, n+1}^{\prime} /\|u\|_{z, n}^{\prime} \leq \exp (-(1-8 \varepsilon) \chi)$ for every $u \in d f^{n}\left(E_{z}^{s}\right)$ and

$$
\exp ((1-8 \varepsilon) \chi) \leq\|d f(v)\|_{z, n+1}^{\prime} /\|v\|_{z, n}^{\prime} \leq(K+6 \varepsilon \chi)
$$

for every $v \in d f^{n}\left(\left(E_{z}^{s}\right)^{\perp}\right)$.

(b) on each $\Lambda_{l}(l \geq 1),\langle\cdot, \cdot\rangle_{z, 0}^{\prime}$ depend on $z$ continuously.

(c) $\|\cdot\| \leq\|\cdot\|_{z, 0}^{\prime} \leq C_{D} \cdot l^{12}\|\cdot\|$ if $z \in \Gamma_{\text {l }}$ where $C_{D}$ is a constant.

(d) For every $w, y \in D\left(f^{n} z, \alpha\right)$,

$$
\left.\| d\left(f_{x}\right)_{w}-d(f)_{x}\right)_{y}\left\|_{z, n, n+1}^{\prime} \leq D\right\| w-y \|_{z . n}^{\prime}
$$

where $x=f^{n} z$ and $\|\cdot\|_{z, n, n+1}^{\prime}$ denote the operator norm with respect to the norms $\|\cdot\|_{z, n}^{\prime}$ and $\|\cdot\|_{z, n+1}^{\prime}$.

(e) $D\left(f^{n} z, \alpha\right) \supset\left\{(v, u) \in d f^{n}\left(E^{s}\right) \oplus d f^{n}\left(\left(E^{s}\right)^{\perp}\right) \mid\|v\|_{z, n}^{\prime} \leq \kappa,\|u\|_{z, n}^{\prime} \leq \kappa\right\}$ where $\kappa=\exp (K+1)$. 
(f) $d f^{n}\left(E_{z}^{s}\right)$ and $d f^{n}\left(\left(E_{z}^{s}\right)^{\perp}\right)$ are orthogonal with respect to $\langle\cdot, \cdot\rangle_{z, n}^{\prime}$.

Proof. Put, for $z \in \Gamma$ and $n \geq 0$,

$$
\begin{aligned}
& \tilde{A}^{s}(z, n)=\sup \left\{\|v\|_{z, n}^{\prime \prime} /\|v\|_{f^{n} z} \mid v \in d f_{z}^{n} E_{z}^{s}\right\}, \\
& \tilde{A}^{t}(z, n)=\sup \left\{\|v\|_{z, n}^{\prime \prime} /\|v\|_{f^{n} z} \mid v \in d f_{z}^{n}\left(\left(E_{z}^{s}\right)^{\perp}\right)\right\}, \\
& \tilde{A}^{\gamma}(z, n)=\left\{\text { angle between } d f_{z}^{n} E_{z}^{s} \text { and } d f_{z}^{n}\left(\left(E_{z}^{s}\right)^{\perp}\right)\right\}^{-1},
\end{aligned}
$$

and

$$
\begin{aligned}
A^{j}(z, n) & =\left(\sup _{k \geq 0} \tilde{A}^{j}(z, k) \exp (-3 \varepsilon \chi k)\right) \cdot \exp (3 \varepsilon \chi n), \quad j=s, t, \gamma, \\
A(z, n) & =4 \cdot A^{\gamma}(z, n) \max \left\{A^{s}(z, n), A^{t}(z, n)\right\} .
\end{aligned}
$$

Then we can see that, if we take a number $c>0$ sufficiently large, the metric $\langle\cdot, \cdot\rangle_{z, n}^{\prime} \equiv(c \cdot A(z, n))^{2}\langle\cdot, \cdot\rangle_{z, n}^{\prime \prime}$ satisfies the conditions in the proposition.

Let us prepare some facts from the invariant manifold theory. Let $E$ be a Euclidian space such that $\operatorname{dim} E=\operatorname{dim} M$. The corresponding inner product and norm, we denote by $\langle\cdot, \cdot\rangle$ and $\|\cdot\|$. We fix an orthogonal decomposition $E=E^{s} \oplus E^{u}$ with $\operatorname{dim} E^{s}=k$, and set $B^{j}(r)=\left\{v \in E^{j} \mid\|v\| \leq r\right\}$ $(j=s, u)$ and $B(r)=B^{s}(r) \times B^{u}(r) \subset E$. Let $D^{2}(B(r), E)$ be the set of $C^{2}$ diffeomorphisms from $B(r)$ into $E$ endowed with $C^{2}$ topology, and let $L(r) \subset D^{2}(B(r), E)$ be the set of linear isomorphisms $h: B(r) \rightarrow E$ which satisfies $h\left(E^{j} \cap B(r)\right) \subset E^{j} \quad(j=s, u)$ and, for $u \in E^{s} \cap B(r)$ and $v \in E^{u} \cap B(r)$, we have

$$
\exp (-K-6 \varepsilon \chi) \leq\|h(u)\| /\|u\| \leq \exp (-(1-8 \varepsilon) \chi)
$$

and

$$
\exp ((1-8 \varepsilon) \chi) \leq\|h(v)\| /\|v\| \leq \exp (K+6 \varepsilon \chi) .
$$

Then we can find a neighborhood $\mathscr{V}$ of the compact subset $L(1)$ in $D^{2}(B(1), E)$ with the following properties:

For any countable family $\mathbf{F}=\left\{f_{i} \in \mathscr{V} \mid i \in \mathbf{Z}\right\}$,

(P1) $V_{n}^{s}(\mathbf{F})=f_{0}^{-1} \circ f_{1}^{-1} \circ \cdots \circ f_{n}^{-1}\left(B^{s}(1) \times\{0\}\right)\left(\right.$ resp. $V_{n}^{u}(\mathbf{F})=f_{-1} \circ \cdots \circ$ $\left.f_{-n}\left(B^{u}(1) \times\{0\}\right)\right)$ is the graph of a $C^{1}$ mapping

$$
\varphi_{n}^{s}(\mathbf{F}): B^{s}(1) \rightarrow B^{u}(1) \quad\left(\operatorname{resp} . \varphi_{n}^{u}(\mathbf{F}): B^{u}(1) \rightarrow B^{s}(1)\right)
$$

with

$$
\left\|d \varphi_{n}^{s}(\mathbf{F})_{x}\right\| \leq(1 / 2) \quad\left(\text { resp. }\left\|d \varphi_{n}^{u}(\mathbf{F})_{x}\right\| \leq(1 / 2)\right),
$$

(P2) $\varphi_{n}^{s}(\mathbf{F})\left(\operatorname{resp} . \varphi_{n}^{u}(\mathbf{F})\right)$ converges toward a $C^{1}$ mapping

$$
\varphi_{\infty}^{s}(\mathbf{F}): B^{s}(1) \rightarrow B^{u}(1) \quad\left(\text { resp. } \varphi_{\infty}^{u}: B^{u}(1) \rightarrow B^{s}(1)\right)
$$


in the $C^{1}$ sense, uniformly in $\mathbf{F} \in \mathscr{V}$ and, moreover,

$$
\begin{aligned}
\operatorname{graph}\left(\varphi_{\infty}^{s}(\mathbf{F})\right)= & V^{s}(\mathbf{F}) \equiv \bigcap_{i=0}^{\infty} f_{0}^{-1} \circ \cdots \circ f_{i}^{-1}(B(1)) \\
& \left(\text { resp. } \operatorname{graph}\left(\varphi_{\infty}^{u}(\mathbf{F})\right)=V^{u}(\mathbf{F}) \equiv \bigcap_{i=1}^{\infty} f_{-1} \circ \cdots \circ f_{-i}(B(1))\right),
\end{aligned}
$$

(P3) for any $w, z \in V^{s}(\mathbf{F})\left(\operatorname{resp} . V^{u}(\mathbf{F})\right)$

$$
\begin{aligned}
\left\|f^{n}(w)-f^{n}(z)\right\| \leq \exp (- & (\chi / 2) n)\|w-z\| \\
& \quad\left(\operatorname{resp} .\left\|f^{-n}(w)-f^{-n}(z)\right\| \leq \exp (-(\chi / 2) n)\|w-z\|\right),
\end{aligned}
$$

(P4) for any $n \geq 0$ and

$$
w, z \in V^{u}(\mathbf{F}) \cap\left(\bigcap_{i=0}^{n} f_{0}^{-1} \circ \cdots \circ f_{n}^{-1}(B(1))\right),
$$

we have

$$
-1 \leq \log \left\{\operatorname{Jac}\left(d f^{(n)} \mid T_{w} V^{u}(\mathbf{F})\right)\right\}-\log \left\{\operatorname{Jac}\left(d f^{(n)} \mid T_{z} V^{u}(\mathbf{F})\right)\right\} \leq 1
$$

where $f^{(n)}=f_{n} \circ \cdots \circ f_{0}$ and $\operatorname{Jac}(\cdot)$ denote the Jacobian with respect to the Riemannian volumes on the domain and the image induced by $\|\cdot\|$.

See [HPS] for (P1), (P2), (P3) and we refer [M, III.3, Lemma 3.2] for (P4). For this $\mathscr{V}$, we can find a positive number $D_{0}$ such that, if $h_{1} \in D^{2}(B(\kappa), E)$ and $h_{2}, h_{3} \in D^{2}(B(2), E)$ satisfy

$$
\left\|h_{1}-\mathrm{id}\right\|_{C^{2}} \leq D_{0}, \quad\left\|h_{3}-\mathrm{id}\right\|_{C^{2}} \leq D_{0} \quad \text { and } \quad\left\|h_{2}-h\right\|_{C^{2}} \leq D_{0}
$$

for some $h \in L(2) \quad\left(\|\cdot\|_{C^{2}}\right.$ denotes the $C^{2}$ norm), then $\left.h_{1}^{-1} \circ h_{2} \circ h_{3}\right|_{B(1)} \in \mathscr{V}$. Let us fix families of metrics $\langle\cdot, \cdot\rangle_{x}^{\prime}$ and $\langle\cdot, \cdot\rangle_{z, n}^{\prime}$ which we constructed for $D=D_{0}$ in Propositions 6 and 7. Then, from the choice of $D_{0}$ and properties (P1)-(P3), we can get the following proposition.

Proposition 8 (Pesin's stable manifold theorem). A. There exist families of $C^{1}$ embedded disks $V_{\Gamma}^{s}(z)(z \in \Gamma)$ with the following properties:

(1) $z \in V_{\Gamma}^{s}(z)$ and $T_{z} V_{\Gamma}^{s}(z)=E_{z}^{s}$,

(2) on each $\Gamma_{l}(l \geq 1), V_{\Gamma}^{s}(z)$ depends on $z$ continuously in the $C^{1}$ sense,

(3) $\limsup \operatorname{sum}_{n \rightarrow \infty}(1 / n) \log d\left(f^{n} w, f^{n} y\right) \leq-(1 / 3) \chi$ for $w, y \in V_{\Gamma}^{s}(z)$.

B. There exists families of $C^{1}$ embedded disks $V_{\Lambda}^{s}(x)$ and $V_{\Lambda}^{u}(x) \quad(x \in \Lambda)$ with the following properties:

(1) $\{x\}=V_{\Lambda}^{s}(x) \cap V_{\Lambda}^{u}(x)$ and $T_{x} V_{\Lambda}^{j}(x)=E_{x}^{j}(j=s, u)$,

(2) on each $\Lambda_{l}(l \geq 1), V_{\Lambda}^{s}(x)$ and $V_{\Lambda}^{u}(x)$ depend on $x$ continuously in the $C^{1}$ sense,

(3) $\lim \sup _{n \rightarrow \infty}(1 / n) \log d\left(f^{n} w, f^{n} y\right) \leq-(1 / 3) \chi$ for $w, y \in V_{\Lambda}^{s}(x)$ and $\lim \sup _{n \rightarrow \infty}(1 / n) \log d\left(f^{-n} w, f^{-n} y\right) \leq-(1 / 3) \chi$ for $w, y \in V_{\Lambda}^{u}(x)$.

Remark. The properties $\mathrm{A}(2)$ and $\mathrm{B}(2)$ are the consequences of the uniform convergence in (P2). 
Before closing this section we prove

Proposition 9. If the Lyapunov exponents $\lambda_{i}(y), i=1,2, \ldots, \operatorname{dim} M$ at $a$ point $y \in \mathscr{R}$ satisfies

$$
\begin{aligned}
\lambda_{1}(y) & \leq \cdots \leq \lambda_{k}(y)<-(1-4 \varepsilon)^{-1} \chi<0<(1-4 \varepsilon)^{-1} \chi<\lambda_{k+1}(y) \\
& \leq \cdots \leq \lambda_{\operatorname{dim} M}(y)
\end{aligned}
$$

then $y$ is contained in $W^{s}\left(\tilde{\Lambda}_{l}\right)$ for some large $l \geq 1$.

Proof. From Proposition 3, there exists $l^{\prime \prime} \geq 1$ such that $y \in \widetilde{\Gamma}_{l^{\prime \prime}}$. This implies $\mu_{y}\left(\Gamma_{l^{\prime \prime}}\right)>0$. On the other hand, by Oseledec's theorem and Lemma 2, we have $\mu_{y}(\Lambda)=1$. Therefore we can find a point $x$ in $\Gamma_{l^{\prime \prime}} \cap \Lambda$ such that a subsequence $\left\{f^{n(i)} y \in \Gamma_{l^{\prime \prime}}\right\}_{i=1}^{\infty}$ converges toward $x$. Since the embedded disks $V_{\Gamma}^{s}(x)$ and $V_{\Lambda}^{u}(x)$ intersect transversally at $x$, from Proposition $8 \mathrm{~A}(2)$, we can find $j \in \mathbf{Z}^{+}$such that $V_{\Lambda}^{u}(x)$ and $V_{\Gamma}^{s}\left(f^{n(j)} y\right)$ intersect transversally at a point $z$. Put $E_{z}^{s}=T_{z} V_{\Gamma}^{s}\left(f^{n(j)} y\right)$ and $E_{z}^{u}=T_{z} V_{\Lambda}^{u}(x)$. Since $z \in V_{\Lambda}^{u}(x)$ and $z \in V_{\Gamma}^{s}\left(f^{n(j)} y\right)$, we can find a constant $l^{\prime} \geq 1$ such that, for $m \in \mathbf{Z}^{+}, n \in \mathbf{Z}^{+}$ and for $u \in d f^{-m}\left(E_{z}^{s}\right)$ and $v \in d f^{-m}\left(E_{z}^{u}\right)$,

$$
\begin{gathered}
\left\|d f^{n} u\right\| \leq l^{\prime} \exp (\varepsilon \chi m) \exp (-\chi n)\|u\|, \\
\left\|d f^{n} v\right\| \geq\left(l^{\prime} \exp (\varepsilon \chi m)\right)^{-1} \exp (\chi n)\|v\|
\end{gathered}
$$

and

(angle between $d f^{-m}\left(E_{z}^{s}\right)$ and $\left.d f^{-m}\left(E_{z}^{u}\right)\right) \geq\left(l^{\prime} \exp (\varepsilon \chi m)\right)^{-1}$.

Since $z \in V_{\Gamma}^{s}\left(f^{n(j)} y\right)$, the point $z$ is regular for $\mu_{y}$. Applying the same procedure which we have used in the proof of Proposition 3 to the decomposition $T_{z} M=E_{z}^{s} \oplus E_{z}^{u}$, we can see that the point $z$ is contained in $\widetilde{\Lambda}_{l}$ for some large l. (Remark that we know, from (4), $\rho^{j}(n)<l^{\prime} \cdot \exp (\varepsilon \chi|n|)$ for $n \leq 0$ in this case.) Therefore we have $y \in W^{s}\left(f^{-n(j)} z\right) \subset W^{s}\left(\tilde{\Lambda}_{l}\right)$.

\section{Construction of ergodic Sinai measures}

Except the last part of this section where we prove the main results, we fix the number $0<\chi<10^{-1}$ and $k \in \mathbf{Z}^{+}$and use the notations in $\S 3$. In this section, we denote, by $\|\cdot\|_{x}^{\prime}$, the metric on $\Lambda$ which we constructed for $D=D_{0}$ in Proposition 6. At each point $x \in \Lambda$, we fix an isometric linear map

$$
\tau_{x}:(E,\|\cdot\|) \rightarrow\left(T_{x} M,\|\cdot\|_{x}^{\prime}\right)
$$

which takes $E^{s}$ and $E^{u}$ to $E_{x}^{s}$ and $E_{x}^{u}$, respectively. Let us consider a countable subset $\Omega$ of the set $\mathscr{C} \equiv\left\{(x, k) \in \Lambda \times \mathbf{Z}^{+} \mid x \in \Lambda_{\exp (\varepsilon \chi k)}\right\}$ satisfying, for every $n \in \mathbf{Z}^{+}$,

$$
\#\{(x, k) \in \Omega \mid k=n\}<\infty .
$$


The "transition matrix" for $\Omega, A_{\Omega}=\left\{a_{q, r}\right\}_{q, r \in \Omega}$, is defined in the following way: for $q=(x, n)$ and $r=(y, m)$,

$$
\begin{array}{ll}
a_{q, r}=1 \quad & \text { if }|n-m| \leq 1 \text { and } \\
& f_{q, r} \equiv \tau_{y}^{-1} \circ \exp _{y}^{-1} \circ f \circ \exp _{x} \circ \tau_{x}: E \nearrow E \\
& \text { is defined on } B(1) \text { and }\left.f_{q, r}\right|_{B(1)} \in \mathscr{V}, \\
a_{q, r}=0 & \text { otherwise. }
\end{array}
$$

The corresponding symbolic dynamics is $\psi: \Sigma_{\Omega} \rightarrow \Sigma_{\Omega}$ :

$$
\Sigma_{\Omega}=\left\{\sigma: \mathbf{Z} \rightarrow \Omega \mid a_{\sigma(i), \sigma(i+1)}=1 \text { for every } i \in \mathbf{Z}\right\}, \quad \psi(\sigma)(i)=\sigma(i+1) .
$$

Then we can define, for $\sigma \in \Sigma_{\Omega}(\sigma(0)=(x, n))$,

$$
\begin{aligned}
& \tilde{V}_{\text {loc }}^{j}(\sigma) \equiv V^{j}\left(\left\{f_{\sigma(i), \sigma(i+1)}\right\}_{i \in \mathbf{Z}}\right) \subset E \quad(\text { see the property }(\mathrm{P} 2) \text { of } \mathscr{V}) \\
& V_{\text {loc }}^{j}(\sigma) \equiv \exp _{x} \circ \tau_{x}\left(\tilde{V}_{\text {loc }}^{j}(\sigma)\right), \quad j=s, u,
\end{aligned}
$$

and

$$
\boldsymbol{\Theta}(\sigma)=V_{\mathrm{loc}}^{s}(\sigma) \cap V_{\mathrm{loc}}^{u}(\sigma) .
$$

Put $V^{s}(\sigma)=\bigcup_{i \geq 0} f^{-i}\left(V_{\mathrm{loc}}^{s}\left(\psi^{i}(\sigma)\right)\right)$ and $V^{u}(\sigma)=\bigcup_{i \geq 0} f^{i}\left(V_{\mathrm{loc}}^{u}\left(\psi^{-i}(\sigma)\right)\right)$. For a subset $Z \subset \Sigma_{\Omega}$, we denote the sets $\bigcup_{\sigma \in Z} V_{\text {loc }}^{j}(\sigma)$ and $\bigcup_{\sigma \in Z} V^{j}(\sigma)$ by $V_{\text {loc }}^{j}(Z)$ and $V^{j}(Z)(j=s, u)$, respectively.

Lemma 10. (1) $\Theta: \Sigma_{\Omega} \rightarrow M$ is continuous.

(2) $f \circ \boldsymbol{\theta}=\boldsymbol{\theta} \circ \psi$,

(3) $V^{s}(\sigma)$ and $V^{u}(\sigma)$ are injectively immersed manifolds and characterized as, for $\sigma \in \Sigma_{\Omega}$,

$$
\begin{aligned}
V^{s}(\sigma) & =\left\{y \in M \mid \limsup _{n \rightarrow \infty}\left\{(1 / n) \log d\left(f^{n} x, f^{n} y\right)\right\} \leq-(1 / 3) \chi\right\} \\
& =\left\{y \in M \mid \limsup _{n \rightarrow \infty}\left\{(1 / n) \log d\left(f^{n} x, f^{n} y\right)\right\} \leq-5 \varepsilon \chi\right\} \\
V^{u}(\sigma) & =\left\{y \in M \mid \limsup _{n \rightarrow \infty}\left\{(1 / n) \log d\left(f^{-n} x, f^{-n} y\right)\right\} \leq-(1 / 3) \chi\right\} \\
& =\left\{y \in M \mid \limsup _{n \rightarrow \infty}\left\{(1 / n) \log d\left(f^{-n} x, f^{-n} y\right)\right\} \leq-5 \varepsilon \chi\right\}
\end{aligned}
$$

where $x=\Theta(\sigma)$.

(4) If $\sigma(0)=\sigma^{\prime}(0)$ for $\sigma, \sigma^{\prime} \in \Sigma_{\Omega}$, then $V_{\text {loc }}^{j}(\sigma)$ and $V_{\text {loc }}^{j}\left(\sigma^{\prime}\right)$ are disjoint or identical $(j=s, u)$.

Proof. (1) and (2) are easy. We prove (3). From the definition of $V^{j}(\mathbf{F})$ in (P2) (§3), we can see that, for $i \in \mathbf{Z}^{+}, f^{-1}\left(V_{\text {loc }}^{s}\left(\psi^{i+1} \sigma\right)\right) \supset V_{\text {loc }}^{s}\left(\psi^{i} \sigma\right)$ and $f\left(V_{\text {loc }}^{u}\left(\psi^{-i-1} \sigma\right)\right) \supset V_{\text {loc }}^{u}\left(\psi^{-i} \sigma\right)$. Therefore, $V^{j}(\sigma)(j=s, u)$ is an injectively immersed manifold. If $y \in M$ satisfies

$$
\limsup _{n \rightarrow \infty}(1 / n) \log d\left(f^{n} x, f^{n} y\right) \leq-5 \varepsilon \chi
$$


then, from Proposition 6(c), we can find a number $N>0$ such that, for $n \geq N$,

$$
f^{n} y \in \exp _{f^{n} x} \circ \tau_{f^{n} x}(B(1)),
$$

and this implies that $y \in V^{s}(\sigma)$. On the other hand, we have, from Proposition $6(\mathrm{c})$ and (P3), that any point $y \in V^{s}(\sigma)$ satisfies (6), replacing $5 \varepsilon \chi$ by $-(1 / 3) \chi$. Similarly, we can get the characterization of $V^{u}(\sigma)$. (4) can be seen from (3).

Proposition 11. There exists a countable subset $\Omega$ of $\mathscr{C}$ with the property (5) and the following property (7): For every $z \in \Lambda$, there exists an element $\sigma \in \Sigma_{\Omega}$ which satisfies $\Theta(\sigma)=z$ and, for every $m \in \mathbf{Z}$,

$$
\exp \{\varepsilon \chi \cdot(n(m)-1)\}<l\left(f^{m}(z)\right) \leq \exp \{\varepsilon \chi \cdot n(m)\}
$$

where $\sigma(m)=(p(m), n(m)) \in \mathscr{C}$.

Proof. For each $n \in \mathbf{Z}$ and $y \in \Lambda_{\exp (\varepsilon \times n)}$, there exist a neighborhood $U_{y, n}^{\prime}$ of $y$ in $\Lambda_{\exp (\varepsilon \chi k)}$ and a continuous vector bundle isomorphism

$$
\tilde{\tau}_{y}: U_{y, n}^{\prime} \times E \rightarrow T_{U_{y, n}^{\prime}} M
$$

such that $\tilde{\tau}_{y}(y, v)=\tau_{y}(v)$ and, for each point $w \in U_{y, n}^{\prime}$, the map

$$
\tilde{\tau}_{y, w} \equiv \tilde{\tau}_{y}(w, \cdot):(E,\|\cdot\|) \rightarrow\left(T_{w} M,\|\cdot\|_{w}^{\prime}\right)
$$

is an isometric linear map which takes $E^{s}$ and $E^{u}$ to $E_{x}^{s}$ and $E_{x}^{u}$, respectively. If we choose a neighborhood $U_{y, n} \subset U_{y, n}^{\prime}$ of $y$ in $\Lambda_{\exp (\varepsilon \chi k)}$ sufficiently small, then we have

$$
\left\|\left.\left(\tilde{\tau}_{y, w}^{-1} \circ \exp _{w}^{-1} \circ \exp _{y} \circ \tau_{y}-\mathrm{id}\right)\right|_{B(\kappa)}\right\|_{C^{2}} \leq D_{0}
$$

for every $w \in U_{y, n}$, and $U_{y, n} \subset \exp _{y} \circ \tau_{y}(B(1))$. Take such $U_{y, n}$ for every $y \in \Lambda_{\exp (\varepsilon \chi n)}$ and every $n \in \mathbf{Z}^{+}$. Since $\Lambda_{\exp (\varepsilon \chi n)}$ is a compact set, we can choose a finite subset $\Omega_{n}$ of $\Lambda_{\exp (\varepsilon \chi n)}$ so that $\left\{U_{p, n} \mid p \in \Omega_{n}\right\}$ covers $\Lambda_{\exp (\varepsilon \chi n)}$. Put $\Omega=\bigcup_{n}\left(\Omega_{n} \times\{n\}\right) \subset \mathscr{C}$. We prove that this set $\Omega$ has the property (7). For a point $z \in \Lambda$, choose $\sigma(m)=(p(m), n(m)) \in \Omega$ for $m \in \mathbf{Z}$ so that

$$
\exp \{\varepsilon \chi \cdot(n(m)-1)\}<l\left(f^{m} z\right) \leq \exp \{\varepsilon \chi \cdot n(m)\}
$$

and $f^{m}(z) \in U_{p(m), n(m)}$. Since

$$
\begin{aligned}
\left.f_{\sigma(m), \sigma(m+1)}\right|_{B(1)}= & \left.\tau_{p(m+1)}^{-1} \circ \exp _{p(m+1)}^{-1} \circ f \circ \exp _{p(m)} \circ \tau_{p(m)}\right|_{B(1)} \\
= & \left(\tilde{\tau}_{p(m+1), f^{m+1}(z)}^{-1} \circ \exp _{f^{m+1}(z)}^{-1} \circ \exp _{p(m+1)} \circ \tau_{p(m+1)}\right)^{-1} \\
& \circ\left(\tilde{\tau}_{p(m+1), f^{m+1}(z)}^{-1} \circ \exp _{f^{m+1}(z)}^{-1} \circ f \circ \exp _{f^{m}(z)} \circ \tilde{\tau}_{p(m), f^{m}(z)}\right) \\
& \left.\circ\left(\tilde{\tau}_{p(m), f^{m} z}^{-1} \circ \exp _{f^{m} z}^{-1} \circ \exp _{p(m)} \circ \tau_{p(m)}\right)\right|_{B(1)},
\end{aligned}
$$

from the choice of the number $D_{0}$, we can see that

$$
\left.f_{\sigma(m), \sigma(m+1)}\right|_{B(1)} \in \mathscr{V}
$$


and, therefore, $a_{\sigma(m), \sigma(m+1)}=1$ for $m \in \mathbf{Z}$. Since $\tau_{p(m)}^{-1} \circ \exp _{p(m)}^{-1} \circ f^{m}(z) \in B(1)$ for every $m \in \mathbf{Z}$, we have $\Theta(\sigma)=z$.

Let us fix a subset $\Omega \subset \mathscr{C}$ with the properties (5) and (7), and put, for $p=(x, n)$,

$$
\Sigma_{p}=\left\{\sigma \in \Sigma_{\Omega} \mid \sigma(0)=p\right\}
$$

and $\Sigma_{p}^{\prime}=\left\{\sigma \in \Sigma_{p} \mid m^{\prime}(\{m \in \mathbf{Z} \mid \sigma(m)=p\})>0\right.$ and $\left.V_{\operatorname{loc}}^{s}(\sigma) \cap \Lambda_{\exp (\varepsilon \chi n)} \neq \varnothing\right\}$. Then we have, from (5) and (7),

$$
\bigcup_{p \in \Omega} \bigcup_{i=0}^{\infty} f^{-i}\left(\Theta\left(\Sigma_{p}^{\prime}\right)\right) \supset \bigcup_{n} \tilde{\Lambda}_{\exp (\varepsilon \chi n)}=\bigcup_{l} \tilde{\Lambda}_{l} .
$$

Let $L_{j} \subset B(1)(j=1,2)$ be a graph of a $C^{1}$ mapping $\varphi_{j}: B^{u}(1) \rightarrow B^{s}(1)$ which satisfy

$$
\left\|d \varphi_{j}(x)\right\| \leq(1 / 2) \quad \text { for } x \in B^{u}(1) .
$$

We define a mapping

by

$$
S\left(L_{1}, L_{2}\right): L_{1} \cap \tilde{V}_{\operatorname{loc}}^{s}\left(\Sigma_{p}^{\prime}\right) \rightarrow L_{2} \cap \widetilde{V}_{\log }^{s}\left(\Sigma_{p}^{\prime}\right)
$$

$$
S\left(L_{1}, L_{2}\right)\left(L_{1} \cap \widetilde{V}_{\mathrm{loc}}^{s}(\sigma)\right)=L_{2} \cap \widetilde{V}_{\mathrm{loc}}^{s}(\sigma) \quad \text { for } \sigma \in \Sigma_{p}^{\prime} .
$$

Lemma 12. (1) $S\left(L_{1}, L_{2}\right)$ is continuous.

(2) $S\left(L_{1}, L_{2}\right)$ is absolutely continuous with respect to the Riemannian volume on $L_{i}$ induced by the norm $\|\cdot\|$. Moreover, there exists a constant $C_{p}>0$ such that

$$
C_{p}^{-1} \leq\left|\operatorname{Jac}\left(S\left(L_{1}, L_{2}\right)\right)(x)\right| \leq C_{p}
$$

for every $x \in L_{1} \cap \tilde{V}_{\text {loc }}^{s}\left(\Sigma_{p}^{\prime}\right)$ for every $L_{1}$ and $L_{2}$ satisfying (9) where $\operatorname{Jac}(\cdot)$ denote the Jacobian.

These properties are called 'absolute continuity of the family of stable manifolds' and, for the proof, we refer [PS] and [KS]. (The original proof is contained in [P1].)

Now let us construct ergodic Sinai measures. We fix $p \in \Omega$ for which $\widetilde{V}_{\mathrm{loc}}^{s}\left(\Sigma_{p}^{\prime}\right)$ has positive Lebesgue measure. Since $\Sigma_{p}^{\prime} \neq \varnothing$, we can find an element $\rho \in \Sigma_{p}^{\prime}$ such that $\psi^{i}(\rho)=\rho$ for some $i \in \mathbf{N}$. Obviously, $\Theta(\rho)$ is a hyperbolic periodic point.' Let us denote, by $\lambda_{0}$, the Riemannian volume on $\widetilde{V}_{\text {loc }}^{u}(\rho)$ induced by the norm $\|\cdot\|$. By Fubini's theorem and Lemma 12, we have

$$
\lambda_{0}\left(\widetilde{V}_{\text {loc }}^{u}(\rho) \cap \widetilde{V}_{\text {loc }}^{s}\left(\Sigma_{p}^{\prime}\right)\right)>0 .
$$

Put $\boldsymbol{\Phi}_{k, p} \equiv\left\{\sigma \in \Sigma_{p}^{\prime} \mid \sigma(-i-k)=\rho(-i)\right.$ for every $\left.i \geq 0\right\}$ for $k \in \mathbf{Z}^{+}$. Then, since $\bigcup_{\sigma \in \Phi_{k, p}} V_{\text {loc }}^{u}(\sigma) \subset f^{k}\left(V_{\text {loc }}^{k}(\rho)\right)$, we can find a finite subset $\Phi_{k, p}^{\prime}$ of $\Phi_{k, p}$ such that $\bigcup_{\sigma \in \Phi_{k, p}} V_{\text {loc }}^{u}(\sigma)$ is the disjoint union of $\left\{V_{\mathrm{loc}}^{u}(\sigma) \mid \sigma \in \Phi_{k, p}^{\prime}\right\}$. We set

$$
\mu_{n}=(1 / n) \sum_{k=0}^{n-1}\left(f^{k} \circ \exp _{x} \circ \tau_{x}\right)_{*}\left(\lambda_{0}\right)
$$


and

$$
\nu_{n}=(1 / n) \sum_{k=0}^{n-1} \sum_{\sigma \in \Phi_{k, p}^{\prime}}\left(\left.\left(f^{k} \circ \exp _{x} \circ \tau_{k}\right)_{*}\left(\lambda_{0}\right)\right|_{V_{\mathrm{loc}}^{u}(\sigma)}\right) .
$$

Then we can find a subsequence $\{n(j)\}_{j=1}^{\infty}$ such that $\mu_{n(j)}$ and $\nu_{n(j)}$ converge to some measures $\mu_{p}$ and $\nu_{p}$ in weak topology, respectively. Obviously, $\mu_{p}$ is $f$-invariant and $\nu_{p}$ is absolutely continuous to $\mu_{p}$. Since $V_{\text {loc }}^{u}\left(\Sigma_{p}\right)$ is a compact set, we have $\operatorname{supp}\left(\nu_{p}\right) \subset V_{\text {loc }}^{u}\left(\Sigma_{p}\right)$.

Lemma 13. $\nu_{p}\left(V_{\mathrm{loc}}^{u}\left(\Sigma_{p}\right)\right)>0$.

Proof. Define a function $\xi_{n}: \widetilde{V}_{\text {loc }}^{u}(\rho) \cap \widetilde{V}_{\text {loc }}^{s}\left(\Sigma_{p}\right) \rightarrow \mathbf{R}$ by

$$
\xi_{n}(x)=(1 / n) \max \left\{\#\{0 \leq i \leq n-1 \mid \sigma(i)=p\} \mid \sigma \in \Sigma_{p}, \Theta(\sigma)=x\right\} .
$$

Then we have

$$
\nu_{n}\left(V_{\mathrm{loc}}^{u}\left(\Sigma_{p}\right)\right) \geq \int \xi_{n} d \lambda_{0}
$$

and, therefore, by the compactness of $V_{\text {loc }}^{u}\left(\Sigma_{p}\right)$, we have

$$
\begin{aligned}
\nu_{p}\left(V_{\mathrm{loc}}^{u}\left(\Sigma_{p}\right)\right) & \geq \liminf _{n \rightarrow \infty} \int \xi_{n} d \lambda_{0} \\
& \geq \int_{\widetilde{V}_{\mathrm{loc}}^{u}(\rho) \cap \widetilde{V}_{\mathrm{loc}}^{s}\left(\Sigma_{p}^{\prime}\right)}\left\{\liminf _{n \rightarrow \infty} \xi_{n}\right\} d \lambda_{0} \\
& >0 . \quad \square
\end{aligned}
$$

Let us call a Borel set $X \subset V_{\text {loc }}^{s}\left(\Sigma_{p}^{\prime}\right)$ (resp. $Y \subset V_{\text {loc }}^{u}\left(\Sigma_{p}\right)$ ) is an $S$-set (resp. a $U$-set) if $X=V_{\text {loc }}^{s}(Z)$ (resp. $Y=V_{\text {loc }}^{u}(Z)$ ) for some subset $Z$ of $\Sigma_{p}$.

Lemma 14. There exists a constant $L_{p}$ such that, for any $S$-set $X$ and $U$-set $Y$, we have

$$
L_{p}^{-1} \leq\left(\nu_{p}(X \cap Y)\right) /\left(\nu_{p}(Y) \times \lambda_{0}^{\prime}\left(X \cap V_{\mathrm{loc}}^{u}(\rho)\right)\right) \leq L_{p}
$$

where $\lambda_{0}^{\prime} \equiv\left(\exp _{x} \circ \tau_{x}\right)_{*} \lambda_{0}$.

Proof. From Lemma 12(1) and the counter part of it for unstable manifolds, it is enough to prove (10) in the case $X$ and $Y$ are compact. Take decreasing sequence of $S$-set $\left\{X_{i}\right\}$ and $U$-set $\left\{Y_{i}\right\}$ such that $\bigcap_{i=0}^{\infty} X_{i}=X, \bigcap_{i=0}^{\infty} Y_{i}=Y$ and that $X_{i}$ is open in $V_{\mathrm{loc}}^{s}\left(\Sigma_{p}^{\prime}\right)$ and $Y_{i}$ is open in $V_{\mathrm{loc}}^{u}\left(\Sigma_{p}\right)$. Then we have

$$
\nu_{p}(Y)=\lim _{i \rightarrow \infty} \liminf _{j \rightarrow \infty} \nu_{n(j)}\left(Y_{i}\right)
$$

and $\nu_{p}(X \cap Y)=\lim _{i \rightarrow \infty} \liminf _{j \rightarrow \infty} \nu_{n(j)}^{\prime}\left(X_{i} \cap Y_{i}\right)$. On the other hand, by (P4) and Lemma 12, we have

$$
\begin{gathered}
\left(e \cdot C_{p}\right)^{-1} \nu_{n(j)}\left(Y_{i}\right) \cdot \lambda_{0}^{\prime}\left(X_{i} \cap V_{\mathrm{loc}}^{u}(\rho)\right) \leq \nu_{n(j)}\left(X_{i} \cap Y_{i}\right) \\
\leq\left(e \cdot C_{p}\right) \nu_{n(j)}\left(Y_{i}\right) \cdot \lambda_{0}^{\prime}\left(X_{i} \cap V_{\mathrm{loc}}^{u}(\rho)\right) .
\end{gathered}
$$

Therefore we have (10) for $L_{p}=e \cdot C_{p}$. 
Lemma 15. For any continuous function $\varphi: M \rightarrow \mathbf{R}$, the time average

$$
\tilde{\varphi}_{+}(x) \equiv \lim _{n \rightarrow \infty}(1 / n) \sum_{i=0}^{n-1} \zeta \circ f^{i}(x)
$$

exists and is constant for $\nu_{p}$-almost every $x \in V_{\mathrm{loc}}^{u}\left(\Sigma_{p}\right)$ and for Lebesgue almost every $x \in V_{\text {loc }}^{s}\left(\Sigma_{p}^{\prime}\right)$.

Proof. For $\alpha \in \mathbf{R}$, the sets

$$
\begin{aligned}
X^{0} & =\left\{x \in V_{\mathrm{loc}}^{s}\left(\Sigma_{p}^{\prime}\right) \mid \tilde{\varphi}_{+} \text {does not exist }\right\}, \\
X_{\alpha}^{+} & =\left\{x \in V_{\mathrm{loc}}^{s}\left(\Sigma_{p}^{\prime}\right) \mid \tilde{\varphi}_{+} \geq \alpha\right\}, \\
X_{\alpha}^{-} & =\left\{x \in V_{\mathrm{loc}}^{s}\left(\Sigma_{p}^{\prime}\right) \mid \tilde{\varphi}_{+}<\alpha\right\}
\end{aligned}
$$

are $S$-sets, and the sets

$$
\begin{aligned}
Y^{0} & =\left\{x \in V_{\mathrm{loc}}^{u}\left(\Sigma_{p}\right) \mid \tilde{\varphi}_{-} \equiv \lim _{n \rightarrow \infty}(1 / n) \sum_{i=0}^{n-1} \varphi \circ f^{-i}(x) \text { does not exist }\right\}, \\
Y_{\alpha}^{+} & =\left\{x \in V_{\mathrm{loc}}^{u}\left(\Sigma_{p}\right) \mid \tilde{\varphi}_{-} \geq \alpha\right\}, \quad Y_{\alpha}^{-}=\left\{x \in V_{\mathrm{loc}}^{u}\left(\Sigma_{p}\right) \mid \tilde{\varphi}_{-}<\alpha\right\}
\end{aligned}
$$

are $U$-sets. Remark that, for $\nu_{p}$-almost every point $x, \tilde{\varphi}_{+}(x)$ and $\tilde{\varphi}_{-}(x)$ exist and coincide. Therefore, by Lemma 14, we can see that the Lebesgue measure of $X^{0}$ is zero, $\nu_{p}\left(Y^{0}\right)=0$ and that, for any $\alpha \in \mathbf{R}$, either

$$
\nu_{p}\left(Y_{\alpha}^{+}\right)=0 \text { and the Lebesgue measure of } X_{\alpha}^{+} \text {is zero }
$$

or

$$
\nu_{p}\left(Y_{\alpha}^{-}\right)=0 \text { and the Lebesgue measure of } X_{\alpha}^{-} \text {is zero. }
$$

These imply the conclusion.

The above lemma implies that there exists an ergodic component $\tilde{\mu}_{p}$ of $\mu_{p}$, to which $\nu_{p}$ is absolutely continuous.

Definition [L]. A measure $\mu$ is said to be absolutely continuous with respect to unstable foliation if, for every measurable partition $\xi$ such that the elements satisfy $\mu$-almost everywhere, $C_{\xi}(x) \subset W^{u}(x)$ and $\mu_{W^{u}(x)}\left(C_{\dot{\xi}}(x)\right)>0$, the family of conditional measures $m_{x}^{\xi}$ on $C_{\xi}(x)$ has the following property: $m_{x}^{\xi}$ is absolutely continuous with respect to $\mu_{W^{u}(x)} \mu$-almost everywhere. $\left(C_{\xi}(x)\right.$ denote the element of $\xi$ which contains $x$ and $\mu_{W^{u}(x)}$ denote the Lebesgue measure on $W^{u}(x) \equiv\left\{y \in M \mid \lim \sup _{n \rightarrow \infty}(1 / n) \log d\left(f^{-n} x, f^{-n} y\right)<0\right\}$.)

Remark. For an ergodic measure $\mu$ whose exponents $\lambda_{i}(\mu)$ satisfy

$$
\begin{aligned}
\lambda_{1}(\mu) & \leq \lambda_{2}(\mu) \leq \cdots \leq \lambda_{k}(\mu)<-(1+\varepsilon) \chi<0<(1+\varepsilon) \chi \\
& <\lambda_{k+1}(\mu) \leq \cdots \leq \lambda_{\operatorname{dim} M}(\mu),
\end{aligned}
$$

$V^{u}(x)$ and $W^{u}(x)$ coincide almost everywhere. (See Lemma 10(3) and remark that we can take the number $\varepsilon$ arbitrary small.) Therefore, we can replace the word ' $W^{u}(x)$ ' by ' $V^{u}(x)$ ' in the above definition. 
Proposition 16. The ergodic measure $\tilde{\mu}_{p}$ has the following properties:

(1) $\tilde{\mu}_{p}$ is absolutely continuous with respect to the unstable foliation.

(2) $\operatorname{supp} \tilde{\mu}_{p}=$ closure $W^{u}(o(\Theta(\rho)))=\operatorname{closure}\left\{W^{u}(o(\Theta(\rho))) \cap W^{s}(o(\Theta(\rho)))\right\}$.

(3) The set of points which are regular for $\tilde{\mu}_{p}$ has positive Lebesgue measure.

Proof. (1) Let $\xi$ be a measurable partition which satisfies $C_{\xi}(x) \subset W^{u}(x)$ and $\mu_{W^{u}(x)}\left(C_{\xi}(x)\right)>0$. Lemma 14 implies that the the absolutely continuous part of $m_{x}^{\xi}(x \in \Lambda)$ with respect to $\mu_{W^{u}(x)}$ does not vanish for $x \in \Theta\left(\Sigma_{p}^{\prime}\right)$. Since the integration of the absolutely continuous part of $m_{x}^{\xi}(x \in \Lambda)$ with respect to $\mu_{W^{u}(x)}$ is invariant under the action of $f$ and since $\tilde{\mu}_{p}$ is ergodic, we can see that $m_{x}^{\xi}$ is absolutely continuous with respect to $\mu_{W^{u}(x)}$ for $\tilde{\mu}_{p}$-almost everywhere.

(2) From the construction of $\nu_{p}$, we have

$$
\tilde{\mu}_{p}\left(\operatorname{closure}\left\{W^{u}(o(\Theta(\rho))) \cap W^{s}(o(\Theta(\rho)))\right\}\right)>0 .
$$

Since $\tilde{\mu}_{p}$ is ergodic, we have

$$
\operatorname{supp} \tilde{\mu}_{p} \subset \operatorname{closure}\left\{W^{u}(o(\Theta(\rho))) \cap W^{s}(o(\Theta(\rho)))\right\} .
$$

On the other hand, since $W^{s}(\Theta(\rho))$ intersects $V_{\text {loc }}^{u}(\sigma)$ for every $\sigma \in \Sigma_{p}$ transversally, and $\operatorname{supp} \tilde{\mu}_{p} \supset V_{\text {loc }}^{u}\left(\sigma_{0}\right)$ for some $\sigma_{0} \in \Sigma_{p}$. Therefore, we have

$$
W^{u}(o(\Theta(\rho))) \subset \text { closure }\left(\bigcup_{n} f^{n}\left(V_{\mathrm{loc}}^{u}\left(\sigma_{0}\right)\right)\right) \subset \operatorname{supp} \tilde{\mu}_{p} .
$$

(3) If a point $x \in V_{\text {loc }}^{s}(\sigma)$ is regular for $\tilde{\mu}_{p}$, then every point in $V_{\text {loc }}^{s}(\sigma)$ is regular for $\mu_{p}$. Therefore the set of all points in $V_{\text {loc }}^{s}\left(\Sigma_{p}^{\prime}\right)$ which are not regular for $\tilde{\mu}_{p}$ is an $S$-set. Since $\nu_{p}$-almost every point is regular for $\tilde{\mu}_{p}$, we can see, from Lemma 14 and Lemma 12(2), that Lebesgue almost every point in $V_{\mathrm{loc}}^{s}\left(\Sigma_{p}^{\prime}\right)$ is regular for $\tilde{\mu}_{p}$.

From (8) and the above proof of Proposition 16(3), we can see that Lebesgue almost every point in $V^{s}\left(\bigcup_{n} \tilde{\Lambda}_{\exp (\varepsilon \chi n)}\right)$ is regular for one of measures $\left\{\tilde{\mu}_{p} \mid V_{\operatorname{loc}}^{s}\left(\Sigma_{p}^{\prime}\right)\right.$ has positive Lebesgue measure $\}$.

Proposition 17. If $\mu$ is an ergodic Sinai measure whose exponents satisfy (11), then there exists $p \in \Omega$ such that $\mu=\tilde{\mu}_{p}$.

Proof. Since $\mu\left(\bigcup_{n \geq 0} \bigcup_{p \in \Omega} f^{-n} \circ \Theta\left(\Sigma_{p}^{\prime}\right)\right)=\mu\left(\bigcup_{l} \tilde{\Lambda}_{l}\right)=1$, we can find $p \in \Omega$ such that $\mu\left(\Theta\left(\Sigma_{p}^{\prime}\right)\right)>0$. Consider a measurable partition $\xi$ such that $C_{\xi}(x) \subset$ $W^{u}(x)$ and $\mu_{W} u_{(x)}\left(C_{\xi}(x)\right)>0 \mu$-almost everywhere and $C_{\xi}(x)=\boldsymbol{\Theta}\left(\Sigma_{p}^{\prime}\right) \cap$ $V_{\text {loc }}^{u}(\sigma)$ for $\sigma \in \Sigma_{p}^{\prime}$ and $x=\Theta(\sigma)$. Since $m_{x}^{\xi}$ is absolutely continuous with respect to Lebesgue measure on $W^{u}(x)$ for $\mu$-almost every $x \in \Theta\left(\Sigma_{p}^{\prime}\right)$. We can see, from Lemma 12, that there exists an $S$-set $X \subset V_{\text {loc }}^{s}\left(\Sigma_{\rho}^{\prime}\right)$ with positive 
Lebesgue measure such that every point in $X$ is generic for $\mu$. From Lemma $15, \mu=\tilde{\mu}_{p}$.

Now let us vary the values of $\chi \in\left(0,10^{-1}\right)$ and $k \in \mathbf{Z}^{+}$. Denote, by $V_{k, \chi}^{s}(x)$, the set $V^{s}(x)$ which we constructed for fixed $\chi$ and $k$. From Proposition 9 and Lemma 10(3), we have

$$
\mathscr{R} \subset W^{s}\left(\bigcup_{k} \bigcap_{n=1}^{\infty} \bigcup_{m=n}^{\infty} \bigcup_{l} \tilde{\Lambda}_{(1 / m), l}^{k}\right) \subset \bigcup_{k} \bigcup_{m=1}^{\infty} V_{k,(1 / m)}^{s}\left(\bigcup_{l} \tilde{\Lambda}_{(1 / m), l}^{k}\right) .
$$

Therefore we can get the 'if part' of Theorem A and Theorem B from the fact that Lebesgue almost every point in $V_{k, \chi}^{s}\left(\bigcup_{l} \widetilde{\Lambda}_{\chi, l}^{k}\right)$ is generic for ergodic Sinai measures. From Propositions 17 and $16(3)$, we can see the 'only if' part of Theorem A. Since ergodic components of Sinai measures are also Sinai measures (cf. [L]) and, from Theorem A, they are at most countably many, we can get Theorem $\mathrm{C}(1) \Rightarrow(2)$. On the other hand, from (12) we can get Theorem $C(2) \Rightarrow(1)$. Proposition D can be seen from Propositions 17 and 16(2).

\section{REFERENCES}

[B] R. Bowen, Equilibrium states and the ergodic theory of Anosov diffeomorphism, Lecture Notes in Math., vol. 470, Springer, New York, 1975.

[GH] J. Guckenheimer and P. Holmes, Nonlinear oscillations, dynamical systems, and bifurcation of vector field, Appl. Math. Sci., vol. 42, Springer, New York, 1986.

[K] A. B. Katok, Lyapunov exponents, entropy and periodic orbits for diffeomorphism, Inst. Hautes Études Sci. Publ. Math. 51 (1980), 137-173.

[KS] A. B. Katok and J. P. Strelcyn, Invariant manifolds, entropy and billiards; smooth maps with singularities, Lecture Notes in Math., vol. 1222, Springer, New York, 1986.

[HPS] M. W. Hirsh, C. C. Pugh, and M. Shub, Invariant manifolds, Lecture Notes in Math., vol. 583, Springer, New York, 1977.

[L] F. Ledrappier, Propriétés ergodique des mesures de Sinaĭ, Inst. Hautes Études Sci. Publ. Math. 59 (1984), 163-188.

[M] R. Mañè, Ergodic theory and differentiable dynamics, Springer-Verlag, New York, 1987.

[O] V. I. Oseledec, A multiplicative ergodic theorem. Ljapunov characteristic numbers for $d y$ namical systems, Trans. Moscow Math. Soc. 19 (1968), 197-231.

[P1] Ya. B. Pesin, Families of invariant manifolds corresponding to nonzero characteristic exponents, Math. USSR-Izv. 10 (1976), no. 6, 1261-1305.

[P2] _ Lyapunov characteristic exponent and smooth ergodic theory, Russian Math. Surveys 32 (1977), no. 4, 55-114.

[PS] C. Pugh and M. Shub, Ergodic attractors, Trans. Amer. Math. Soc. 312 (1989).

[R1] D. Ruelle, Ergodic theory of differentiable dynamical systems, Inst. Hautes Études Sci. Publ. Math. 50 (1979), 27-58.

[R2] - Sensitive dependence on initial conditions and turbulent behavior of dynamical systems, Ann. New York Acad. Sci. 316 (1979), 408-416.

Department of Mathematics, Faculty of Sciences, Kyoto University, Kyoto 606, JAPAN 